\title{
Number Needed to Treat for Recurrent Events
}

\section{Richard J Cook*}

Department of Statistics and Actuarial Science, University of Waterloo, 200 University Avenue West, Waterloo, ON, Canada

\begin{abstract}
Many clinical investigators find the Number Needed to Treat (NNT) an appealing measure of treatment effect and use it routinely in reporting the results of randomized trials. It is most easily computed and interpreted for trials with binary responses, but attempts have been made to compute NNT-like measures for recurrent event outcomes. We discuss methodological issues concerning the construction of NNT-like measures of treatment effect based on recurrent event outcomes. Rate and mean functions are used to develop nonparametric estimates of NNT-like measures of treatment effect for recurrent events in terms of the number of individuals to be treated to expect to prevent a $k^{\text {th }}$ event, and simply to prevent any event. Parametric analyses facilitate the derivation of alternative measures and associated estimates. Applications to a trial of patients with cystic fibrosis are given for illustration. In settings where mortality rates are non-negligible, joint NNT-like measures for the recurrent event and survival processes are required and these are discussed.
\end{abstract}

Keywords: Clinical trials; Expected number of events; Mean function; Rate function; Recurrent events

\section{Introduction}

The number needed to treat (NNT) has been used increasingly often by clinical researchers interested in expressing the consequences of different treatment strategies. Laupacis et al. [1] pointed out limitations in making use solely of relative measures of treatment effect such as relative risks, relative risk reductions and odds ratios, primarily because they do not reflect the control event rate and hence the practical consequences of using an effective treatment in clinical terms. The absolute risk difference conveys the difference in risk of events under two treatment strategies (use of the experimental treatment or use of the control). Inversion of the risk difference gives an estimate of the number of patients that would be required to treat with the experimental treatment rather than the control before one could expect, on average, to be fewer events in the scenario in which patients were treated with the experimental treatment.

The number needed to treat has been criticized by several authors [2], but nevertheless, there is a strong apparent interest in reporting NNT-like measures for the analysis of recurrent event data [3] and attempts to do this have caused much discussion [4]. We consider the associated issues and propose approaches that can be adopted to meet these objectives.

The remainder of this paper is organized as follows. In section 2 we define some notation and functions pertaining to recurrent event processes and discuss concepts relevant to the formulation of NNTlike measures for recurrent events. In section 3, we discuss issues in constructing NNT-like measures in settings with recurrent events. An application is given for illustrative purposes involving data from a trial of patients with cystic fibrosis. Extensions to deal with recurrent events with a terminal event such as death are discussed in section 4 . Some general remarks are made in section 5 .

\section{Some Functions for Modelling Recurrent Event Data}

Consider a randomized clinical trial in which individuals are to be followed over time in order to record the occurrence of clinical events of interest. Follow-up may be planned over $(0, C]$ and we let $C_{i}^{\dagger}$ denote a random time representing when individual $i$ is lost to follow-up; we therefore observe individual $i$ over $\left(0, C_{i}\right]$ where, $C_{i}=\min \left(C_{i}^{\dagger}, C\right)$. Let $t=0$ denote a common time origin taken to be the time of randomization. Let $0<T_{i 1}<T_{i 2}<\cdots$ denote the times of events occurring for individual $i, i=1, \ldots, m$. If $T_{i k} \leq C_{i}$ then the $k^{\text {th }}$ event is observed and we let $X_{i k}=\min \left(T_{i k}, C_{i}\right)$ and $\delta_{i k}=I\left(X_{i k}=T_{i k}\right)$ be the censoring indicator. It is also helpful to define $Y_{i k}(s)=I\left(s \leq T_{i k}\right), Y_{i}(s)=I\left(s \leq C_{i}\right), \bar{Y}_{i k}(s)=Y_{i}(s) Y_{i k}(s)$, and $d N_{i k}(s)=I\left(T_{i k}=s\right)$.

Let $P\left(T_{i k} \leq t\right)=F_{k}(t)$ denote the cumulative marginal distribution function for the time to the $k^{\text {th }}$ event and let

$$
h_{k}(t)=\lim _{\Delta t \rightarrow 0} \frac{P\left(t \leq T_{i k}<t+\Delta t \mid t \leq T_{i k}\right)}{\Delta t},
$$

denote the corresponding hazard function; note $F_{k}(t)=\exp \left(-H_{k}(t)\right)$ where $H_{k}(t)=\int_{0}^{t} h_{k}(s) d s$. The Kaplan-Meier estimate of $F_{k}(t)$ is the nonparametric maximum likelihood estimate given by

$$
\hat{F}_{k}(t)=\prod_{(0, t]}\left(1-d \hat{H}_{k}(s)\right)
$$

Where, $d \hat{H}_{k}(s)=d \bar{N}_{. k}(s) / \bar{Y}_{. k}(s)$ where $d \bar{N}_{. k}(s)=\sum_{i=1}^{m} \bar{Y}_{i k}(s) d N_{i k}(s)$ and $\bar{Y}_{. k}(s)=\sum_{i=1}^{m} \bar{Y}_{i k}(s)[5,6]$.

The random variable $N_{i}(t)=\sum_{j=1}^{\infty} I\left(T_{i j} \leq t\right)$ is a count of the number of events occurring over $(0, t]$ for individual $i$, where $I(\cdot)$ is an indicator function such that $I(A)=1$ if $A$ is true and $I(A)=0$ otherwise. When viewed as a stochastic process over time, $\left\{N_{i}(s), 0 \leq s\right\}$ is a right-continuous counting process. Let $\Delta N_{i}(t)=N_{i}\left(t+\Delta t^{-}\right)-N_{i}\left(t^{-}\right)$ denote the number of events occurring over $[t, t+\Delta t)$ for individual $i$ and $\quad d N_{i}(t)=\lim _{\Delta \downarrow t 0} \Delta N_{i}(t)$ indicate whether an event occurred at $t$ for individual $i$. Figure 1 contains a schematic indicating the relationship between the event times, censoring time and counts.

${ }^{*}$ Corresponding author: Richard J Cook, Department of Statistics and Actuaria Science, University of Waterloo, 200 University Avenue West, Waterloo, Ontario, Canada, E-mail: rjcook@uwaterloo.ca

Received April 10, 2013; Accepted May 07, 2013; Published May 11, 2013

Citation: Cook RJ (2013) Number Needed to Treat for Recurrent Events. J Biomet Biostat 4: 167. doi:10.4172/2155-6180.1000167

Copyright: (c) 2013 Cook BJ. This is an open-access article distributed under the terms of the Creative Commons Attribution License, which permits unrestricted use, distribution, and reproduction in any medium, provided the original author and source are credited. 


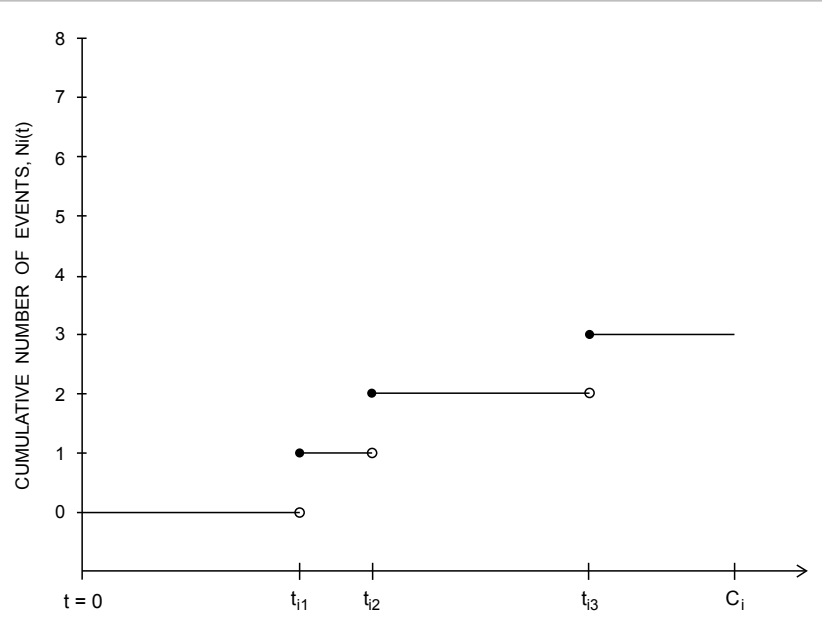

Figure 1: Realization of a counting process and associated notation.

Let $H_{i}(t)=\left\{N_{i}(s), 0<s<t\right\}$ denote the history of the process for individual $i$ at time $t$, which captures the number and times of events from 0 to $t^{-}$. The intensity function of the recurrent event process is the instantaneous conditional probability of an event occurring at $t$ given the history, and is written as

$$
\lambda\left(t \mid H_{i}(t)\right)=\lim _{\Delta t \rightarrow 0} \frac{P\left(\Delta N_{i}(t)=1 \mid H_{i}(t)\right)}{\Delta t}
$$

If two events cannot occur at the same time, then this intensity completely specifies the event process.

We let $d \bar{N}_{i}(t)=Y_{i}(t) d N_{i}(t)$ to indicate that an event was observed at time $t$ for individual $i$. Under the assumption of conditionally independent and non-informative censoring [7], the conditional probability that " $n_{i}$ events occur at times $t_{i 1}<\cdots<t_{i n_{i}}$ " over $\left(0, C_{i}\right]$ for individual $i, i=1, \ldots, m$, is

$$
\prod_{i=1}^{m}\left\{\prod_{j=1}^{n_{i}} \lambda\left(t_{i j} \mid H_{i}\left(t_{i j}\right)\right) \cdot \exp \left\{\int_{0}^{\infty} Y_{i}(u) \lambda\left(u \mid H_{i}(u)\right) d u\right\}\right\} .
$$

While intensity-based models are useful when interest lies in understanding the dynamic aspects of a point process, marginal features such as mean or rate functions are more appealing when analysing data with a view to treatment comparisons in clinical trials. The mean function for example, defined by $\mu(t)=E\left\{N_{i}(t)\right\}$, gives the expected number of events observed over $(0, t]$ per individual. The rate function $\rho(t)=d \mu(t) / d t$ is the derivative of this mean function and so reflects how the rate of events changes over time; we often write $\rho(t) d t=d \mu(t)=E\left\{d N_{i}(t)\right\}$ informally and as a consequence express the mean function as $\mu(t)=\int_{0}^{t} \rho(s) d s$. For Poisson processes, the intensity function is a rate function and so analyses based on rate functions can be likelihood-based under Poisson assumptions. In this case the log-likelihood becomes

$$
\sum_{i=1}^{m}\left\{\sum_{j=1}^{n_{i}} \log \rho\left(t_{i j}\right)-\int_{0}^{\infty} Y_{i}(u) \rho(u) d u\right\} .
$$

If parametric modelling is of interest, then the rate function may be indexed by a $p \times 1$ parameter vector $\theta$ and written as $\rho(t ; \theta)$. In this case we obtain a score vector from equation 1 as $U(\theta)=\sum_{i=1}^{m} U_{i}(\theta)$, where

$$
U_{i}(\theta)=\int_{0}^{\infty} Y_{i}(s) \frac{\partial \log \rho(s ; \theta)}{\partial \theta}\left\{d N_{i}(s)-\rho(s ; \theta) d s\right\} .
$$

If $\hat{\theta}$ is the solution to $U(\theta)=0$, then

$$
\sqrt{m}(\hat{\theta}-\theta) \sim \operatorname{MVN}_{p}\left(0, A^{-1}(\theta) B(\theta) A^{-1}(\theta)\right)
$$

where $A(\theta)=E\left\{-\partial U_{i}(\theta) / \partial \theta^{\prime}\right\}$ and $B(\theta)=E\left\{U_{i}(\theta) U_{i}^{\prime}(\theta)\right\}$. If the recurrent event process is a Poisson process then $A(\theta)=B(\theta)$, but equation 2 is a general result which holds for mixed Poisson processes, for example.

Nonparametric estimation of rate and mean functions is carried out by thinking of $d \mu(s)=\rho(s) d s$ as a quantity of interest and considering the estimating equation for $d \mu(s)$ as

$$
U(s)=\sum_{i=1}^{m} Y_{i}(s)\left\{d N_{i}(s)-d \mu(s)\right\}=0 \quad 0 \leq s,
$$

The solution to $U(s)=0$ is simply $d \hat{\mu}(s)=d N .(s) / Y .(s)$ where $d N .(s)=\sum_{i=1}^{m} Y_{i}(s) d N_{i}(s)$ is the total number of event observed at time $s$ and $Y .(s)=\sum_{i=1}^{m} Y_{i}(s)$ is the total number of individuals under observation at time $s$. The nonparametric estimate of the mean function is then

$$
\hat{\mu}(t)=\int_{0}^{t} d \hat{\mu}(s)
$$

and is called the Nelsen-Aalen estimate [8]. A robust variance for $\sqrt{m}(\hat{\mu}(t)-\mu(t))$ derived by Lawless and Nadeau [9] is

$$
\operatorname{var}\{\sqrt{m}(\hat{\mu}(t)-\mu(t))\}=m \cdot \sum_{i=1}^{m} \int_{0}^{t} \int_{0}^{t} \frac{Y_{i}(u)}{Y .(u)} \frac{Y_{i}(v)}{Y .(v)} \operatorname{cov}\left\{d N_{i}(u), d N_{i}(v)\right\},
$$

and they recommend its use to provide protection against extraPoisson variation. The sample estimate

$$
\widehat{\operatorname{var}}\{\sqrt{m}(\hat{\mu}(t)-\mu(t))\}=m \sum_{i=1}^{m}\left\{\int_{0}^{t} \frac{Y_{i}(u)}{Y .(u)}\left[d N_{i}(u)-\frac{d \bar{N} .(u)}{Y .(u)}\right]\right\}^{2},
$$

can be used to construct confidence intervals or conduct tests of hypotheses.

\section{NNT for Recurrent Event Analyses}

\section{NNT for the $k^{\text {th }}$ Event}

The rationale for using the number needed to treat as a measure of treatment effect is to place the consequences of using the experimental treatment over the control in a context with which clinicians can relate. It is easy to understand that using one treatment strategy over another will lead to a different expectation in terms of the number of events, but in the traditional context of binary responses one can equivalently think of the expected difference in the number of patients that will be affected since each individual may or may not experience an event.

We consider a simple controlled trial in which patients are randomized to receive either a control therapy or an experimental treatment with equation 1 probability. Despite the complex nature of data in trials involving recurrent events, it is common to base treatment comparisons simply on the probability of being "event-free". When all individuals are followed for the same duration of time this can be done simply based on a binary response indicating that an event did or did not occur over the common time period. With variable follow-up however, the use of binary responses is not appropriate and methods which accommodate censoring are required.

Altman and Andersen [10] describe how to compute the number 
needed to treat based on a time to event outcome. Here we generalize this notion to deal with the times to the occurrences of a series of events in the spirit of the marginal graphical approach of Pepe and Cai [11]. Specifically, the number needed to treat to prevent the $k^{\text {th }}$ event is a function of time and is given by $\eta_{k}(t)=\left[F_{0 k}(t)-F_{1 k}(t)\right]^{-1}$ where $F_{j k}(t)$ is the cumulative distribution function for the $k^{\text {th }}$ event in group $j$, $j=0,1$. Then the number needed to treat to prevent the kth event is then estimated as

$$
\hat{\eta}_{k}(t)=\frac{1}{\hat{F}_{0 k}(t)-\hat{F}_{1 k}(t)},
$$

Where, $\hat{F}_{j k}(t)$ is the Kaplan-Meier estimate of $F_{j k}(t)$. In practise, investigators should specify a small number of time points at which this statistic should be computed. Confidence intervals for this number needed to treat can be obtained from Greenwood's formula [6].

\section{NNT for recurrent events-nonparametric analyses}

In the context of recurrent event outcomes, one can chose to summarize the consequences of using one treatment over another in terms of the number of patients affected as discussed in the previous section. Alternatively, if events are of primary interest, one may view it as important to reduce the occurrence of events irrespective of whether they are affecting a new individual or are a secondary event in an individual who already experienced an event. The latter setting is often of interest in health economic analyses where events incur costs and it is desirable to reduce their numbers.

With a sample of size $m$, the expected number of events in the entire sample that will be experienced over $(0, t]$ is $m \mu(t)$. Therefore, if we consider a distinct sample of $m$ patients, the difference in the expected number of events for patients given the treatment versus the control would be $m\left[\mu_{0}(t)-\mu_{1}(t)\right]$, where $\mu_{j}(t)$ is the mean function for individuals in group $j, j=0,1$. Setting this equation 1 to one and solving for $m$ gives the number needed to treat to prevent one recurrent event by time $t$, under treatment versus control conditions, and we write this as $v(t)=\left[\mu_{0}(t)-\mu_{1}(t)\right]^{-1}$. The nonparametric estimate of this is obtained by replacing the mean functions by the Nelson-Aalen estimates giving

$$
\hat{v}(t)=\frac{1}{\hat{\mu}_{0}(t)-\hat{\mu}_{1}(t)}
$$

Note that when treatment effects are strong or the expected number of events in the control arm is large, $\hat{v}(t)$ may be less than one, implying that even if one patient were treated with the experimental treatment rather than the control with sufficient follow-up one would expect to prevent one event. Although this may at first seem odd compared to the usual NNT analysis, it simply indicates that treatment is performing substantially better than the control at time $t$ in that the difference in the expected number of events is greater than one. Again, it is sensible to have some particular time points in mind to assess the $\hat{v}(t)$. The robust variance estimates of Lawless and Nadeau [9] should be used when constructing confidence intervals for $\mu_{0}(t)-\mu_{1}(t)$ which can be inverted to give confidence intervals for $\hat{v}(t)$.

\section{NNT for recurrent events-parametric analyses}

Analyses of recurrent events are frequently based on a time homogeneous Poisson model in epidemiology and clinical trials. In this case the rate function is constant so $\rho(t)=\rho$ and the maximum likelihood estimate of $\rho$ is $\bar{\rho}=\sum_{i=1}^{m} N_{i}\left(C_{i}\right) / \sum_{i=1}^{m} C_{i}$. This is often called the "events-per-person-years" analysis because this estimated rate is simply the total number of events divided by the total (person-years) of observation in the sample. It is a widely used statistic in epidemiology where it plays a central role in tabulating disease incidence rates and computing standardized mortality ratios. Under this model, since the rate is constant over time, $E\{N(t)\}=\rho t$, and the parametric analogue to (Equation 6) is

$$
\bar{v}^{p}(t)=\frac{1}{\left(\bar{\rho}_{0}-\bar{\rho}_{1}\right) t}
$$

Where, $\rho_{0}$ and $\rho_{1}$ denote the event rates in the control and experimental arms respectively and the tilde denotes the corresponding estimate.

Note that if $\rho(s ; \theta)=\rho=\theta$ then

$$
U_{i}(\theta)=\int_{0}^{\infty} Y_{i}(s) \theta^{-1}\left\{d N_{i}(s)-\theta d s\right\}=\theta^{-1}\left\{N_{i}\left(C_{i}\right)-\theta C_{i}\right\}
$$

and so $-\partial U_{i}(\theta) / \partial \theta=\theta^{-2} N_{i}\left(C_{i}\right)$. Therefore $A(\theta)=E\left\{-\partial U_{i}(\theta) / \partial \theta\right\}$ is

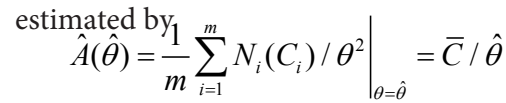

Where, $\bar{C}=\sum_{i=1}^{m} C_{i} / m$ and $B(\theta)=E\left\{U_{i}(\theta) U_{i}^{\prime}(\theta)\right\}$ is estimated by

$$
\hat{B}(\hat{\theta})=\left.\frac{1}{m} \sum_{i=1}^{m} \theta^{-2}\left\{N_{i}\left(C_{i}\right)-\theta C_{i}\right\}^{2}\right|_{\theta=\hat{\theta}}
$$

So

$$
\begin{aligned}
& \widehat{\operatorname{asv} \operatorname{ar}}\{\sqrt{m}(\hat{\theta}-\theta)\}=\left[\frac{1}{m} \sum_{i=1}^{m} N_{i}\left(C_{i}\right) / \hat{\theta}^{2}\right]^{-1}\left[\frac{1}{m} \frac{1}{\hat{\theta}^{2}} \sum_{i=1}^{m}\left(N_{i}\left(C_{i}\right) / \hat{\theta} C_{i}\right)^{2}\right]\left[\frac{1}{m} \sum_{i=1}^{m} N_{i}\left(C_{i}\right) / \hat{\theta}^{2}\right]^{-1} \\
& =m \sum_{i=1}^{m}\left(N_{i}\left(C_{i}\right)-C_{i} \hat{\theta}\right)^{2} / C^{2} .
\end{aligned}
$$

It may also be of interest to estimate how long one would have to treat one patient in order to expect to see one less event under the experimental treatment compared to the control therapy. Therefore one could set $\left(\rho_{0}-\rho_{1}\right) t=1$ and solve for $t$ to get the "time-needed-totreat" $(\tau)$ as $\tau=\left[\rho_{0}-\rho_{1}\right]^{-1}$ which we can estimate by

$$
\bar{\tau}=\frac{1}{\bar{\rho}_{0}-\bar{\rho}_{1}},
$$

and this would give us an estimate of the time needed to treat one patient, on average, in order to prevent one adverse recurrent event. Again, if the difference between $\bar{\rho}_{0}$ and $\bar{\rho}_{1}$ is large enough, then this may be less than one unit of time.

\section{Illustration with application to a cystic fibrosis trial}

Here we consider data from a trial of cystic fibrosis patients in which children were randomized to receive a purified recombinant form of the human enzyme DNase I, called rhDNase, or placebo $[12,13]$. There were 324 and 321 individuals randomized to the placebo and rhDNase arms, respectively. Those randomized to the experimental treatment received it daily and the remaining patients were administered a placebo; patients and their physicians did not know which treatment (rhDNase or placebo) they were receiving. Follow-up was for approximately 169 days, and the occurrences of exacerbations over the study period were recorded for each individual. Individuals experienced as many as five exacerbations. 

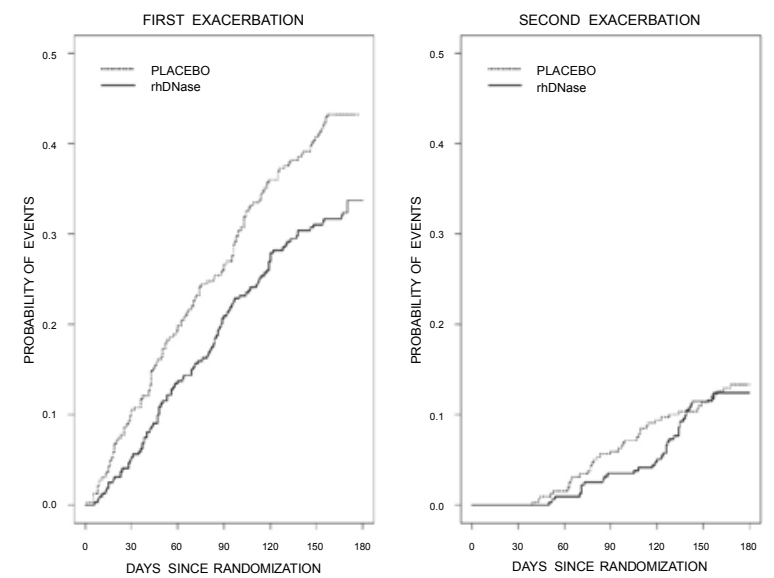

Figure 2: Estimated cumulative distribution functions for the first (left panel) and second (right panel) exacerbation for each treatment group.

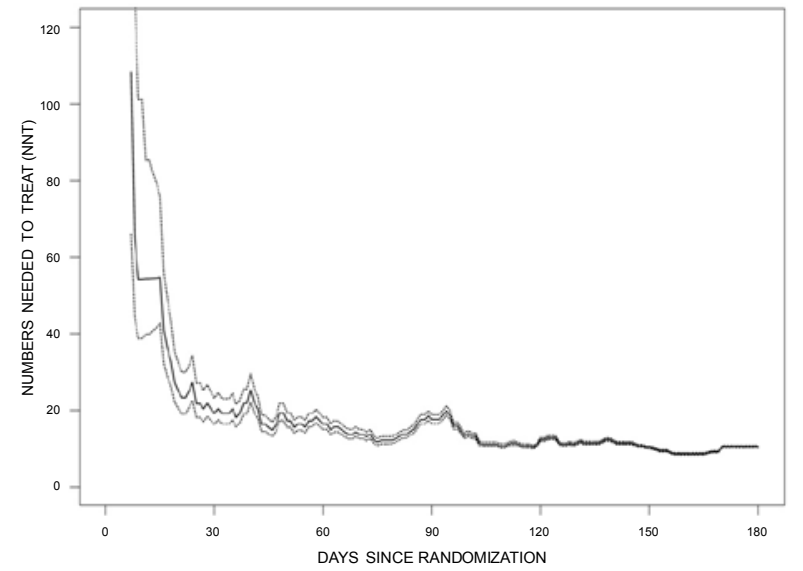

Figure 3: Number needed to treat estimates for the first exacerbation (solid line). Dotted line indicates the $95 \%$ confidence interval bands.

Figure 2 gives the Kaplan-Meier estimates of the proportion of individuals with at least one exacerbation (left panel) and at least two exacerbations (right panel) as a function of time since randomization. It can be seen that while an estimated $43 \%$ of placebo treated individuals have at least one exacerbation by 180 days, only approximately $13 \%$ of individuals on placebo treatment experience a second exacerbation. Moreover, the rhDNase treatment does not have a strong effect in delaying the second exacerbation. The plot of the nonparametric estimate of the number needed to treat to prevent the first event is in figure 3 from which it can be seen that very few individuals must be treated with rhDNase to expect to save an individual from experiencing their first exacerbation $(\hat{\eta}(180)=10.5 ; 95 \% \mathrm{CI}: 10.2,10.8)$. To expect to prevent one second event, the estimate is that $\hat{\eta}_{2}(180)=111.9$ individuals must be treated with rhDNase rather than placebo $(95 \% \mathrm{CI}: 99.9,127.2)$.

A central objective of the study was to compare the two treatment groups in terms of exacerbation occurrence. Figure 4A (left panel) contains plots of the Nelson-Aalen estimates of the mean number of exacerbations by treatment group. Figure 4B (right panel) contains the number needed to treat estimates over time corresponding to (Equation 6). We find $\hat{v}(180)=6.8210(95 \%$ CI: $6.4197,7.2758)$. The parametric analysis yielded estimates $\bar{\rho}_{0}=0.0038$ and $\hat{\rho}_{1}=0.0029$ for the placebo and rhDNase treated patients respectively yielding an estimate of $\hat{v}^{p}(180)=6.0183$ from (Equation 7); 95\%CI:3.1938,52.0483, which is in close agreement with the nonparametric estimate.

\section{NNT for Recurrent and Terminal Events}

\section{NNT and competing risks}

Before discussing issues pertaining to recurrent and terminal events, we first consider the simpler problem associated with a threestate model suitable when individuals are at risk of a non-fatal event and death. Here state 0 may represent being "alive and event-free", state 1 may represent being alive with event, and state 2 represents death. If $Z_{i}(s)$ denotes the state occupied by individual $i$ at time $s$, we let

$$
q_{k}(t)=\lim _{\Delta t \rightarrow 0} \frac{P\left(Z_{i}\left((t+\Delta t)^{-}\right)=k \mid Z_{i}\left(t^{-}\right)=0\right)}{\Delta t}
$$

denote the transition intensity for $0 \rightarrow k$ transitions, $k=1$, 2. The cumulative transition intensity is denoted $Q_{k}(t)=\int_{0}^{t} q_{k}(s) d s$ and we sometimes write $d Q_{k}(t)=q_{k}(t) d t$. The probability of a $0 \rightarrow k$ transition occurring at time $s$ and is given by $d Q_{k}(s) \exp \left(-\left[Q_{1}(s)+Q_{2}(s)\right]\right)$. The probability of this event happening at any time over the interval $(0, t]$ is obtained from the cumulative incidence function

$$
F_{k}^{d}(t)=\int_{0}^{t} d Q_{k}(s) \exp \left(-\left[Q_{1}(s)+Q_{2}(s)\right]\right)
$$

Where, the superscript $d$ indicates that this is a cumulative subdistribution function relevant when individuals face a competing risk of death [14].

An event-free survival analysis involves modelling the distribution of the time in state 0 . Let $E$ denote the time that state zero is left and $F^{e}(t)=P(E \leq t)$ be the corresponding cumulative distribution function. Then

$$
F^{e}(t)=F_{1}^{d}(t)+F_{2}^{d}(t)
$$

so the probability of experiencing the event or death can be decomposed into the two causes of failure. These may then be used to compute NNT-like quantities which can be interpreted together.
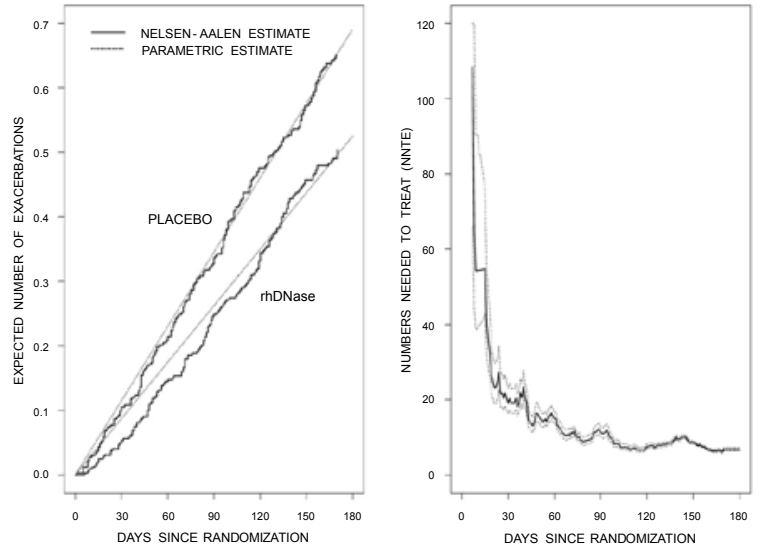

Figure 4: Nelson-Aalen estimates and corresponding parametric (time homogeneous) estimates of the cumulative mean functions (left panel) for placebo and rhDNase treated individuals, respectively, and the number needed to treat for all events (right panel). 
Let $D_{i}$ denote the time of death for individual $i$ and let the "survivor" function be $S(t)=P\left(D_{i} \geq t\right)$.

In a two-sample problem the number needed to treat to expect to prevent a death by time $t$ is $n(t)=\left[\left(1-S_{0}(t)\right)-\left(1-S_{1}(t)\right)\right]^{-1}$ where $S_{j}(t)$ is the survivor function for individuals in group $j, j=0,1$. With this number of patients one can also compute the expected number of nonfatal events prevented as

$$
n(t)\left[F_{01}^{d}(t)-F_{11}^{d}(t)\right]
$$

where $F_{j 1}^{d}(t)$ is the cumulative incidence function for the intermediate event in group $j, j=0,1$.

We let $\bar{Y}_{i}(t)=Y_{i}(t) I\left(D_{i} \geq t\right)$ indicate that individual $i$ is alive and on study at time $t$ and let $d N_{i}^{\dagger}(s)=1$ if individual $i$ dies at time $s$ and be zero otherwise. The Kaplan-Meier estimate of $S(t)$ is $\quad \hat{S}(t)=\prod_{(0, t]}\left(1-d \hat{H}^{\dagger}(u)\right) \quad$ where $\quad d \hat{H}^{\dagger}(u)=d \bar{N}_{.}^{\dagger}(u) / \bar{Y}(t) \quad$ where $d \bar{N}_{.}^{\dagger}(u)=\sum_{i=1}^{m} \bar{Y}_{i}(u) d \bar{N}_{i}^{\dagger}(u)$ and $\bar{Y}(t)=\sum_{i=1}^{m} \bar{Y}_{i}(u)$.

\section{Recurrent and terminal events}

Recurrent events are frequently of interest in settings where patients are at high risk for a different type of event which terminates the recurrent event process. Examples include studies of skeletal complications among patients with cancer metastatic to bone [15], transplant studies where graft rejection episodes may be terminated by total graft rejection [16], and chronic obstructive pulmonary disease (COPD) where patient may experience multiple exacerbations over time but may also die [3]. Figure 5 contains a multistate diagram reflecting this setting. Some clinical trials involving these complex outcomes have endeavoured to compute the number needed to treat and we discuss issues and strategies here.

Cook and Lawless [7] defined a modified rate function which gave the expected number of events at time $u$ conditional on survival time $u$, $0<u$; this is denoted by $d \mu^{*}(u)=E\left\{d N_{i}(u) \mid D_{i} \geq u\right\}$. Then the marginal expected number of events over an interval $(0, t]$, accounting for the possible realization of the terminating event precluding subsequent recurrent events, is the survival adjusted mean function

$$
\mu^{*}(t)=E\left\{N_{i}(t)\right\}=\int_{0}^{t} S(u) d \mu^{*}(u) .
$$

This can be estimated by replacing the unknown quantities with their empirical estimates where $S(u)$ is replaced with the Kaplan Meier estimate $\hat{S}(t)$ and $d \mu^{*}(u)$ is estimated naturally by

$$
d \mu^{*}(u)=\frac{\sum_{i=1}^{m} D_{i}(u) d N_{i}(u)}{\sum_{i=1}^{m} \bar{D}_{i}(u)},
$$

which is simply the number of events observed at time $u$ divided by the number at risk at time $u$. It may be tempting to adapt (Equation 6) and define

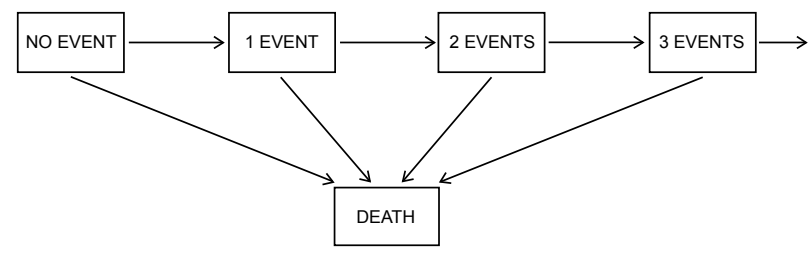

Figure 5: Multistate diagram for recurrent and terminal events.

$$
\hat{v}^{*}(t)=\frac{1}{\hat{\mu}_{0}^{*}(t)-\hat{\mu}_{1}^{*}(t)}
$$

as the number needed to treat to prevent one recurrent event adjusting, or accounting, for the terminal event, death. This is a too simplistic way of exploring the consequences of treatment in this case since one way a treatment may reduce the expected number of recurrent events is by increasing the mortality rate. One must therefore jointly consider the consequences of treatment adoption for both the recurrent event and the terminal event (e.g. survival). We describe two such approaches in the following section.

\section{An Approach when recurrent events are of secondary interest}

The number of individuals needed to treat to expect to save one life over $(0, t]$ is $n(t)=\left[\hat{F}_{0}^{d}(t)-\hat{F}_{1}^{d}(t)\right]^{-1}$. As a consequence of treating this many individuals, one would expect to prevent

$$
n(t)\left(\mu_{0}^{*}(t)-\mu_{1}^{*}(t)\right)
$$

events.

\section{An approach when recurrent events are of primary interest}

One may compute the number needed to treat to prevent one event as before as

$$
v(t)=\frac{1}{\mu_{0}^{*}(t)-\mu_{1}^{*}(t)} .
$$

With this number treated, one would expect to save $v(t)\left[\left(1-S_{0}(t)\right)-\left(1-S_{1}(t)\right)\right]$ lives. Of course in some cases there may be a beneficial treatment effect on the recurrent event process and a detrimental effect on the mortality, so care must be taken in choosing how to express these effects.

\section{Discussion}

In this article we have considered strategies for adapting NNT-like measures for the analysis of more complex life history responses. We consider recurrent events, competing risks and recurrent and terminal events. The latter two situations raise challenges in that two different types of events may both be important and it is essential to consider them together. As in the case with simple binary responses, the idea of computing NNT-like measures is to express treatment effects in terms that clinical researchers may relate to more naturally. It is possible that in more complex settings NNT-like measures may not be suitable.

Because these statistics are based on absolute differences they are natural when examining economic implications of any health policy decisions. They have played a useful role, for example, in considering whether effective treatments with known costs and adverse outcome profiles should be used [17]. They can also be used in concert with measures like the number needed to harm when considering potentially toxic therapies or high risk interventions.

\section{Acknowledgements}

This research was supported by grants from the Natural Sciences and Engineering Research Council of Canada (RGPIN 155849) and the Canadian Institutes for Health Research (FRN 13887). Richard Cook is a Canada Research Chair in Statistical Methods for Health Research.

\section{References}

1. Laupacis A, Sackett DL, Roberts RS (1988) An assessment of clinically usefu measures of the consequences of treatment. N Engl J Med 318: 1728-1733. 
2. Hutton JL (2009) Number needed to treat and number needed to harm are not the best way to report and assess the results of randomised clinical trials. $\mathrm{Br} \mathrm{J}$ Haematol 146: 27-30.

3. Calverley PM, Anderson JA, Celli B, Ferguson GT, Jenkins C, et al. (2007) Salmeterol and fluticasone propionate and survival in chronic obstructive pulmonary disease. N Engl J Med 356: 775-789.

4. Halpin DM (2005) Evaluating the effectiveness of combination therapy to prevent COPD exacerbations: The value of NNT analysis. Int J Clin Pract 59: 1187-1194.

5. Kalbfleisch JD, Prentice RL (2002) The Statistical Analysis of Failure Time Data. (2ndedn) John Wiley and Sons, New York, USA.

6. Lawless JF (2003) Statistical Models and Methods for Lifetime Data. (2ndedn), John Wiley and Sons, Hoboken, New Jersey, USA.

7. Cook RJ, Lawless JF (2007) The Statistical Analysis of Recurrent Events Springer, New York, USA.

8. Nelson W (1995) Confidence Limits for Recurrence Data-Applied to Cost or Number of Product Repairs. Technometrics 37: 147-157.

9. Lawless JF, Nadeau C (1995) Some simple robust methods for the analysis of recurrent events. Technometrics 37: 158-168.

10. Altman DG, Andersen PK (1999) Calculating the number needed to treat for trials where the outcome is time to an event. BMJ 319: 1492-1495.
11. Pepe MS, Cai J (1993) Some graphical displays and marginal regression analyses for recurrent failure times and time dependent covariates. J Am Stat Assoc 88: 811-820.

12. Fuchs $\mathrm{HJ}$, Borowitz DS, Christiansen DH, Morris EM, Nash ML, et al. (1994) Effect of aerosolized recombinant human DNase on exacerbations of respiratory symptoms and on pulmonary function in patients with cystic fibrosis. The Pulmozyme Study Group. N Engl J Med 331: 637-642.

13. Therneau TM, Hamilton SA (1997) rhDNase as an example of recurrent event analysis. Stat Med 16: 2029-2047.

14. Crowder MJ (2001) Classical Competing Risks. Chapman and Hall/CRC, London, UK.

15. Hortobagyi GN, Theriault RL, Porter L, Blayney D, Lipton A, et al. (1996) Efficacy of pamidronate in reducing skeletal complications in patients with breast cancer and lytic bone metastases. Protocol 19 Aredia Breast Cancer Study Group. N Engl J Med 335: 1785-1791.

16. Cole EH, Cattran DC, Farewell VT, Aprile M, Bear RA, et al. (1994)A comparison of rabbit antithymocyte serum and OKT3 as prophylaxis against renal allograft rejection. Transplantation 57: 60-67.

17. Sinclair JC, Cook RJ, Guyatt GH, Pauker SG, Cook DJ (2001) When should an effective treatment be used? Derivation of the threshold number needed to treat and the minimum event rate for treatment. J Clin Epidemiol 54: 253-262. 